\title{
Nonlocal effective parameters of multilayered metal-dielectric metamaterials
}

\author{
A. V. Chebykin, ${ }^{1}$ A. A. Orlov, ${ }^{1}$ C. R. Simovski, ${ }^{1,2}$ Yu. S. Kivshar, ${ }^{1,3}$ and P. A. Belov ${ }^{1,4}$ \\ ${ }^{1}$ National Research University of Information Technologies, Mechanics, and Optics (ITMO), St. Petersburg 197101, Russia \\ ${ }^{2}$ School of Electric and Electronic Engineers, Aalto University, Aalto 00076, Finland \\ ${ }^{3}$ Nonlinear Physics Center, Research School of Physics and Engineering, Australian National University, Canberra ACT 0200, Australia \\ ${ }^{4}$ Queen Mary College, University of London, Mile End Road, London E1 4NS, United Kingdom \\ (Received 4 July 2012; revised manuscript received 3 September 2012; published 14 September 2012)
}

\begin{abstract}
We consider multilayered metal-dielectric metamaterials composed of alternating nanolayers of two types and calculate the components of their effective dielectric permittivity tensors as functions of both frequency and wave vector. We demonstrate that such structures can be described as strongly nonlocal uniaxial effective media, and we analyze how the nonlocal permittivity tensor components are related to other manifestations of strong spatial dispersion in such structures, and how the resonance of permittivity depends on the propagation direction.
\end{abstract}

DOI: 10.1103/PhysRevB.86.115420

PACS number(s): 78.67.Pt, 78.20.Bh

\section{INTRODUCTION}

Multilayered metamaterials (MLMMs), which can be defined as one-dimensional structures of two periodically alternating optically thin layers with sufficient optical contrast between them, are known to have unusual electromagnetic properties, being promising for many applications, especially in the optical frequency range. Optical MLMMs have layer thickness as small as a few tens of nanometers, and the dielectric layers alternate with silver or gold layers having the thickness of the same order. Such MLMMs can transport subwavelength images, ${ }^{1-4}$ and this effect makes them promising for nanolithography. ${ }^{5}$ Also, MLMM-based optical cloaks have been proposed (see, e.g., Refs. 6 and 7). In a certain frequency range, such metal-dielectric MLMMs can be modeled as media with the hyperbolic dispersion surfaces in the space of wave vectors. Such media are usually characterized by an uniaxial permittivity tensor whose axial and transverse components have different signs and therefore these media are called indefinite materials. ${ }^{8}$ With indefinite materials used as substrates or/and superstrates of nanoemitters such as quantum dots or nanoantennas it is possible to obtain huge values for the Purcell factor. ${ }^{9-14}$

The study of MLMMs was probably initiated in the classical paper by Rytov ${ }^{15}$ who derived the dispersion equations for TMand TE-polarized waves and also analyzed the corresponding eigenmodes. An attempt to interpret the refraction indices and wave impedances of these eigenmodes in terms of effective material parameters resulted in retrieved values of $\varepsilon$ and $\mu$ which were characterized by Rytov as physically meaningless parameters since they violated the causality and passivity limitations even in the case when both alternating layers were dielectric and had subwavelength thickness. Rytov concluded that the local effective medium concept can be applied to MLMMs only with finite accuracy and only in some special cases when the quasistatic model of MLMMs is also applicable.

A more advanced theory of MLMMs was developed in Ref. 16. This effective medium model generalized the quasistatic model taking into account the retardation effects of the first order with respect to the optical thickness of a layer. However, this theory was concentrated mainly on the normal propagation, and the limits of its validity remained not well defined. Later, it was shown ${ }^{17,18}$ that the spatial dispersion related to surface plasmon polaritons excited at metal-dielectric interfaces of such structures plays a key role in optical properties of MLMMs. Spatial dispersion implies that the effective permittivity tensor components depend on the wave vector. ${ }^{19,20}$ The existence of strong spatial dispersion effects were revealed for different types of metamaterials, e.g., for arrays of split-ring resonators ${ }^{21}$ and wire metamaterials, ${ }^{22}$ and it is also inherent to MLMMs. New physics brought to multilayered structures by nonlocality includes such effects as emission control, ${ }^{13,14}$ single polarization beam-splitting, ${ }^{26}$ and nonlocal transformation optics. ${ }^{23}$ Since the effective material parameters proposed in Refs. 15 and 16 did not take into account the effects of strong spatial dispersion which actually present in MLMMs, in order to describe them correctly one needs to generalize the homogenization theory taking into account nonlocal effects.

A nonlocal homogenization theory that results in the tensorial permittivity $\overline{\bar{\varepsilon}}$ calculated for all possible values of the wave vector $\mathbf{k}$ and frequency $\omega$ was suggested in Ref. 24. Possible bianisotropic and magnetic responses of the effective medium are comprised, respectively, in the first- and second-order terms of the k-power expansion of $\overline{\bar{\varepsilon}}(\mathbf{k})$. When this $\overline{\bar{\varepsilon}}$ is complemented by additional boundary conditions, it allows solving a boundary value problem for a half space and even for finite-thickness layers of the medium. Unlike other homogenization models, this theory does not imply any approximation. It is a strict approach based on the exact knowledge of microscopic fields in an infinite regular lattice composed of electromagnetically linear and reciprocal dielectric or conducting inclusions. However, for a majority of lattices this homogenization procedure is numerical. In other words, as a rule the components of the nonlocal tensor $\overline{\bar{\varepsilon}}$ cannot be derived analytically beyond certain approximations, and they have to be simulated numerically. To our knowledge, only the case of MLMMs due to its geometrical simplicity allows the exact analytical calculation of $\overline{\bar{\varepsilon}}$. This calculation was described in our recent work ${ }^{25}$ and presented there as an iterative set of rigorous expressions explicitly depending on $\omega$ and $\mathbf{k}$. To compute the components of the nonlocal permittivity tensor, these expressions require performing some 


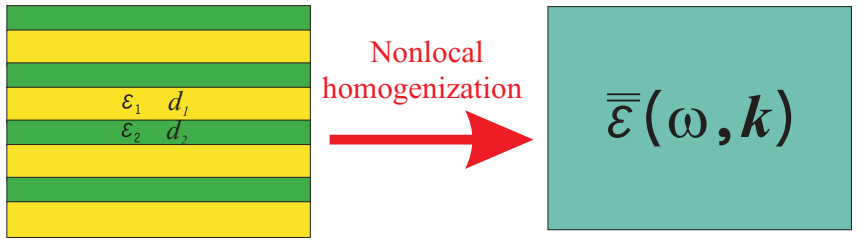

FIG. 1. (Color online) Original structure and its homogenized representation.

matrix algebra. In this paper, we make the next step toward the closed-form expressions. However, these expressions turned out to be very involved, and for some components of $\overline{\bar{\varepsilon}}$ it is reasonable to keep their matrix form.

Thus, this paper aims to analyze the dependencies of the tensor $\overline{\bar{\varepsilon}}$ on $\omega$ and $\mathbf{k}$ and to find a link between these dependencies and the effects of strong spatial dispersions observed in MLMMs; this analysis extends further the results of our earlier publications. ${ }^{25,26}$ A study of eigenmodes of multilayered metamaterials considered in this paper and corresponding field distributions can be found, for example, in the recent Ref. 27.

\section{QUASISTATIC AND NONLOCAL EFFECTIVE MEDIUM MODELS}

In the static limit, an infinite multilayered structure composed of two alternating layers with thicknesses $d_{1}, d_{2}$ and permittivities $\varepsilon_{1}, \varepsilon_{2}$ can be considered as a uniaxial medium having the following permittivity tensor:

$$
\overline{\bar{\varepsilon}}_{\mathrm{loc}}=\left(\begin{array}{ccc}
\varepsilon_{\perp} & 0 & 0 \\
0 & \varepsilon_{\|} & 0 \\
0 & 0 & \varepsilon_{\|}
\end{array}\right)
$$

where

$$
\begin{gathered}
\varepsilon_{\|}=\frac{\varepsilon_{1} d_{1}+\varepsilon_{2} d_{2}}{d_{1}+d_{2}}, \\
\varepsilon_{\perp}=\left(\frac{\varepsilon_{1}^{-1} d_{1}+\varepsilon_{2}^{-1} d_{2}}{d_{1}+d_{2}}\right)^{-1} .
\end{gathered}
$$

This model is employed for the optical frequency region, and it results in the representation of a metal-dielectric MLMM as a local indefinite medium since the permittivity of metals is negative in this range (more exactly, it has a negative real part). However, in Ref. 26 it was revealed that such a description of plasmonic MLMMs is not adequate. The first-order corrections of the quasistatic model obtained in Ref. 16 are not helpful in this case since the spatial dispersion in such structures is strong. Even if the dependence $\overline{\bar{\varepsilon}}(\mathbf{k})$ can be replaced by a power series, the series convergence is not rapid enough to be restricted by second- or third-order terms. Moreover, in the case of strong spatial dispersion the dependence $\overline{\bar{\varepsilon}}(\mathbf{k})$ can be resonant [i.e., in the lossless case the function $\overline{\bar{\varepsilon}}(\mathbf{k})$ can have singularities].

Similar to our previous work, ${ }^{25}$ we choose the coordinate system in such a way that the axis $x$ is normal to the layers, and the Bloch wave with the wave vector $\mathbf{k}$ propagates in the plane $(x, y)\left(k_{z}=0\right)$. Then, a homogenized MLMM (see Fig. 1) can be described by the permittivity tensor: ${ }^{25}$

$$
\overline{\bar{\varepsilon}}(\omega, \mathbf{k})=\left(\begin{array}{ccc}
\varepsilon_{x x}(\omega, \mathbf{k}) & \varepsilon_{x y}(\omega, \mathbf{k}) & 0 \\
\varepsilon_{y x}(\omega, \mathbf{k}) & \varepsilon_{y y}(\omega, \mathbf{k}) & 0 \\
0 & 0 & \varepsilon_{z z}(\omega, \mathbf{k})
\end{array}\right)
$$

From the reciprocity condition, we have $\varepsilon_{x y}(\omega, \mathbf{k})=$ $\varepsilon_{y x}(\omega, \mathbf{k})$, so that there are four independent components of $\overline{\bar{\varepsilon}}$. In the Appendix we present the rigorous equations for calculating these four scalar values.

A quite unexpected result of our earlier study ${ }^{25}$ is the presence of the nonzero value of the off-diagonal components $\varepsilon_{x y}=\varepsilon_{y x}$. It means that in order to transform the permittivity tensor to a diagonal form, one has to rotate the coordinate system $X Y Z$ around the axes $z$. The tensor becomes diagonal; i.e., the medium behaves as a uniaxial medium when the optical axis $x^{\prime}$ is tilted to the normal axis $x$ by a certain angle $\theta$. This angle depends on both frequency $\omega$ and propagation direction. For any $\omega$ it vanishes for both normal $k_{y}=0$ and in-plane $k_{x}=0$, and it is maximal for nearly bisector propagation $\left(k_{y}=k_{x}\right)$. The nonzero value of $\theta$ is an important parameter characterizing the spatial dispersion effect.

Another feature of the spatial dispersion is the nonequivalence of two components $\varepsilon_{y y} \neq \varepsilon_{z z}$ both referring to the plane of layers. If the spatial dispersion effects are negligible both these components would be equivalent and equal to the transversal permittivity of an effective uniaxial medium (whose optical axis would be then normal to the layers). In the quasistatic limit $D \equiv d_{1}+d_{2} \rightarrow 0$ (more exactly when $k_{0} D \rightarrow 0$, where $k_{0}=\omega / c$ is the free space wave number), the nonlocal permittivity tensor (4) numerically turns into Eqs. (1)-(3). This tendency is pointwise (not uniform for all k).

We have checked our calculations of $\overline{\bar{\varepsilon}}(\omega, \mathbf{k})$ in the following way. Eigenmodes in an arbitrary anisotropic material described by the permittivity tensor $\overline{\bar{\varepsilon}}$ are solutions of known dispersion equations. For TE waves this dispersion equation is known, ${ }^{19}$

$$
k_{x}^{2}+k_{y}^{2}=\varepsilon_{z z}\left(\frac{\omega}{c}\right)^{2} .
$$

For TM waves we have the following equation: ${ }^{19}$

$\left(\varepsilon_{x x} \varepsilon_{y y}-\varepsilon_{x y}^{2}\right)\left(\frac{\omega}{c}\right)^{2}-\varepsilon_{y y} k_{y}^{2}-\varepsilon_{x x} k_{x}^{2}-2 k_{x} k_{y} \varepsilon_{x y}=0$.

For an arbitrary MLMM, the rigorous dispersion equations $^{15}$ are the following (for TE and TM waves, respectively):

$$
\begin{aligned}
\Delta_{\mathrm{TE}}= & \sin \left(k_{x 1} d_{1}\right) \sin \left(k_{x 2} d_{2}\right)\left(k_{x 1}^{2}+k_{x 2}^{2}\right)+2 k_{x 1} k_{x 2}\left[\cos \left(k_{x} D\right)\right. \\
& \left.-\cos \left(k_{x 1} d_{1}\right) \cos \left(k_{x 2} d_{2}\right)\right]=0, \\
\Delta_{\mathrm{TM}}= & \sin \left(k_{x 1} d_{1}\right) \sin \left(k_{x 2} d_{2}\right)\left(k_{x 1}^{2}+k_{x 2}^{2}\right)+2 k_{x 1} k_{x 2}\left[\cos \left(k_{x} D\right)\right. \\
& \left.-\cos \left(k_{x 1} d_{1}\right) \cos \left(k_{x 2} d_{2}\right)\right]=0 .
\end{aligned}
$$

Substituting these expressions for the components of $\overline{\bar{\varepsilon}}(\omega, \mathbf{k})$, we find that Eqs. (5) and (6) are equivalent to Eqs. (7) and (8), respectively. Dispersion diagrams of Ref. 26 were obtained by using (8), and for metal-dielectric MLMMs with permittivities $\varepsilon_{1}=4.6$ and $\varepsilon_{2}=1-\lambda^{2} / \lambda_{p}^{2}$, where 


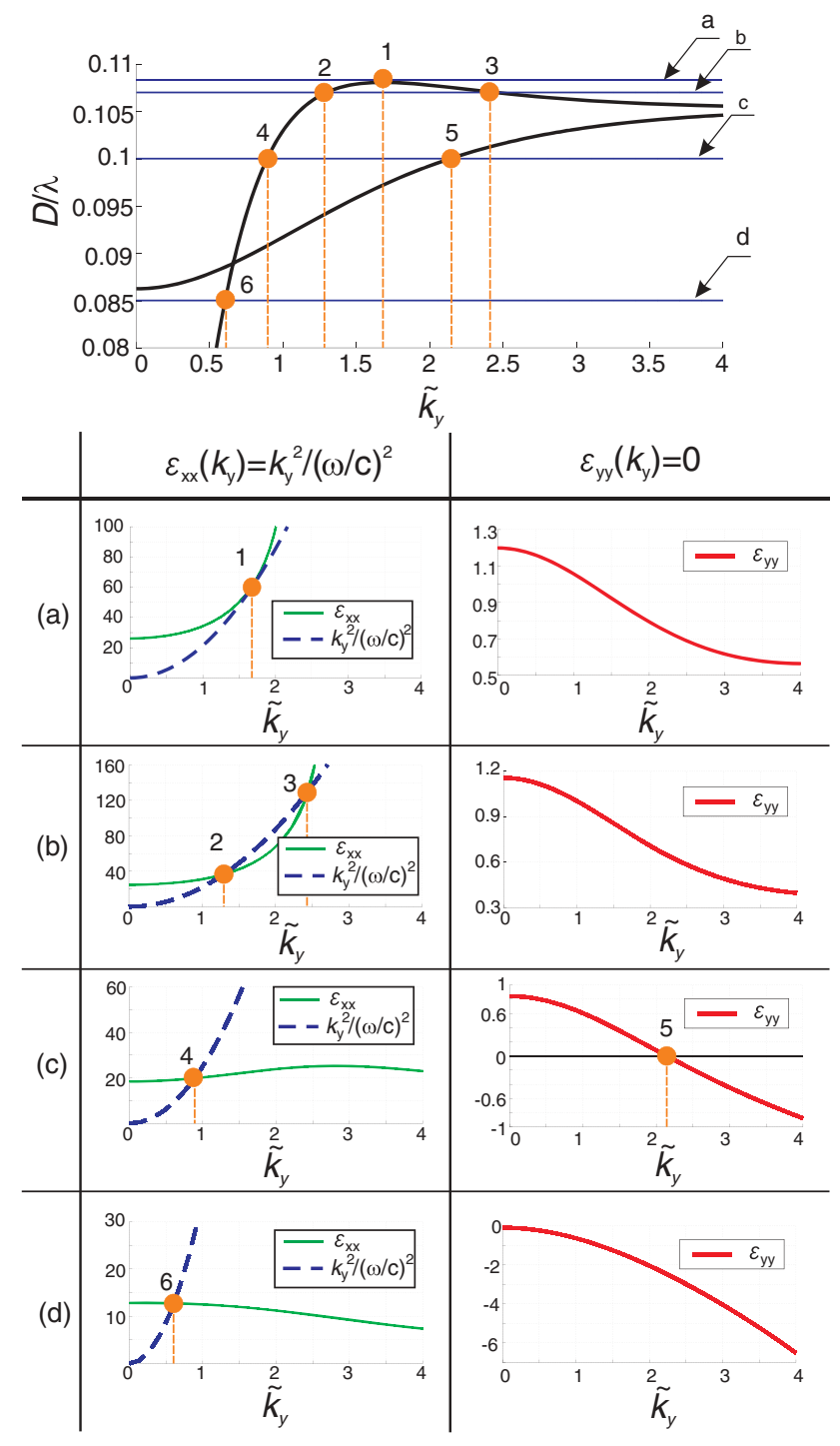

FIG. 2. (Color online) Dispersion diagram of MLMMs and graphical solutions of the dispersion equations (10) for $d_{1}=1.5 d_{2}$ : (a) $D / \lambda=0.108$, (b) $D / \lambda=0.107$, (c) $D / \lambda=0.1$, and (d) $D / \lambda=$ 0.085 .

$\lambda_{p}=250 \mathrm{~nm}$. The dispersion diagrams we discuss are calculated by solving Eq. (6), and they turned out to be exactly the same as those obtained in Ref. 26.

\section{DISPERSION AND NONLOCAL PERMITTIVITY}

We consider the special case of the in-plane propagation $k_{x}=0$ when $\varepsilon_{x y}=0$. Then Eq. (8) simplifies to become

$$
\varepsilon_{y y}\left[\varepsilon_{x x}\left(\frac{\omega}{c}\right)^{2}-k_{y}^{2}\right]=0,
$$

and it is equivalent to the system of two dispersion equations,

$$
\varepsilon_{y y}\left(k_{y}\right)=0, \quad \varepsilon_{x x}\left(k_{y}\right)\left(\frac{\omega}{c}\right)^{2}-k_{y}^{2}=0 .
$$

The first equation in the system (10) corresponds to longitudinal modes, and the second equation, to transverse modes.

In Fig. 2 (top) we show the dispersion curves of the structure with the same values of $\varepsilon_{1}$ and $\varepsilon_{2}$ as above and thicknesses $d_{1}=1.5 d_{2}$ (namely, $d_{1}=37.5 \mathrm{~nm}$ and $d_{2}=25 \mathrm{~nm}$ ). On the frequency axis, we mark four example points and obtain six values of the normalized wave number $\tilde{k}_{y}=k_{y} D / \pi$ corresponding to the selected frequencies. These results can be also obtained by the graphical solutions of the system (10), and we illustrate them with the plots in Figs. 2(a)-2(d). It is also interesting to analyze the selected cases of the values of $\varepsilon_{x x}$ and $\varepsilon_{y y}$ which correspond to these six dispersion states (the dispersion state is a pair of $\omega, \mathbf{k}$ ).

At the normalized frequency $\omega_{a} \equiv D / \lambda=0.108$, we have $\varepsilon_{x x} \approx 60$ and $\varepsilon_{y y} \approx 0.85$. The ratio $\varepsilon_{x x} / \varepsilon_{y y} \approx 70$ is high but it turns out not to be sufficiently large for strong spatial dispersion at this frequency. There is only one wave which in the lossless approximation has zero group velocity (point 1). The presence of such a wave can be explained as a competition of the forward and backward SPP modes. At the normalized frequency $\omega_{b} \equiv D / \lambda=0.107$, we have $\varepsilon_{x x} \approx 40$ and $\varepsilon_{y y} \approx 0.95$ (point 2 ). Then $\varepsilon_{x x} / \varepsilon_{y y} \approx 45$ and anisotropy is again not sufficient for the spatial dispersion. However, at point 3 we have $\varepsilon_{x x} \approx 130$ and $\varepsilon_{y y} \approx 0.6$. Then $\varepsilon_{x x} / \varepsilon_{y y} \approx 220$, and this amazing anisotropy turns out to be large enough for the manifestation of spatial dispersion. Point 3 corresponds to the backward wave which exists in spite of all positive components of the permittivity and permeability. This wave clearly originates from the medium discreteness, and it is the feature of strong spatial dispersion. This strong spatial dispersion corresponds to the large ratio $\varepsilon_{x x} / \varepsilon_{y y}>200$ which is hardly compatible with the concept of a continuous medium.

The strong spatial dispersion is observed also at the normalized frequency $\omega_{c} \equiv D / \lambda=0.1$ where at point 4 we have $\varepsilon_{x x} \approx 20$ and $\varepsilon_{y y} \approx 0.05$. This dispersion state corresponds to the usual (forward transverse) wave. However, at the same frequency there appears point 5 , where $\varepsilon_{x x} \approx 24$ and $\varepsilon_{y y}=0$. The anisotropy in the last case is infinitely large, and the wave corresponding to point 5 is longitudinal. The presence of the longitudinal wave in the lattice is obviously a feature of strong spatial dispersion. ${ }^{21}$ Finally, at the frequency $\omega_{d} \equiv D / \lambda=0.085$ (point 6 ) where $\varepsilon_{x x} \approx 14$ and $\varepsilon_{y y} \approx-0.4$, we have a rather modest ratio $\left|\varepsilon_{x x} / \varepsilon_{y y}\right| \approx 35$, and there are no features of the strong spatial dispersion (only one forward transverse wave).

The plots presented in Fig. 3 for the case $d_{2}=1.5 d_{1}$ are analogous of the plots in Fig. 2. Here at $\omega_{a} \equiv D / \lambda=0.115$ (point 1) $\varepsilon_{x x} \approx-5$ and $\varepsilon_{y y}=0$. The wave is not only longitudinal, it is also backward, and this is the frequency where the spatial dispersion is strong. At $\omega_{b} \equiv D / \lambda=0.099$ (point 2) we have $\varepsilon_{x x} \approx 120$ and $\varepsilon_{y y} \approx-1$. Then $\left|\varepsilon_{x x} / \varepsilon_{y y}\right| \approx$ 120 , and this ratio turns out to be not sufficient to justify the spatial dispersion. At this frequency, there exists only one transverse forward wave.

For the case of a thin metal $\left(d_{1}=1.5 d_{2}\right)$, we reveal that in the vicinity of the plasmon resonance frequency the dispersion diagram possesses a fine structure shown in Fig. 4. The branch for which two waves coexist at the same frequency demonstrates even more unusual behavior: Three waves instead of one can be excited at the same frequency at the single branch. Assuming that there exists also the second branch, we have four waves in the region of the fine structure of MLMM dispersion. 

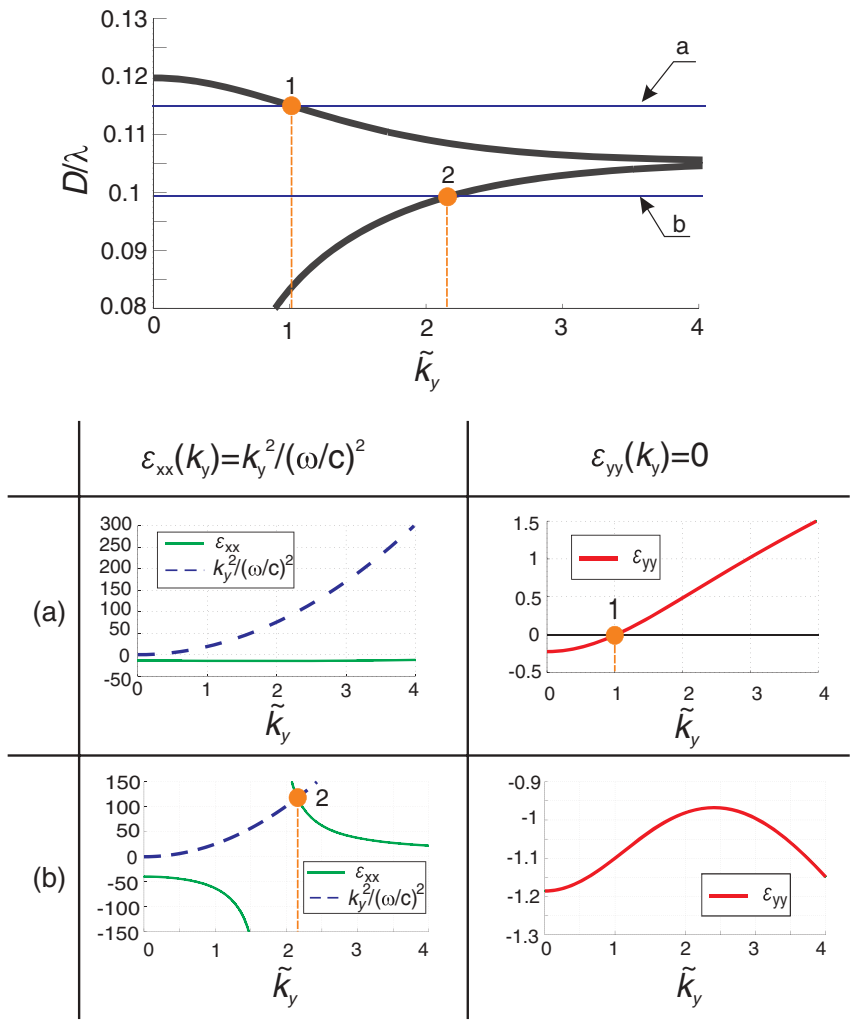

FIG. 3. (Color online) Dispersion diagram of MLMMs and graphical solutions of dispersion equations (10) for $d_{2}=1.5 d_{1}$ and (a) $D / \lambda=0.115$, (b) $D / \lambda=0.099$.

From this analysis, one can conclude that for the structure under study there is a direct correspondence between its anisotropy and the spatial dispersion. Of course, the existence of the spatial dispersion effect follows from the dependence of the permittivity tensor on the wave vector. However,
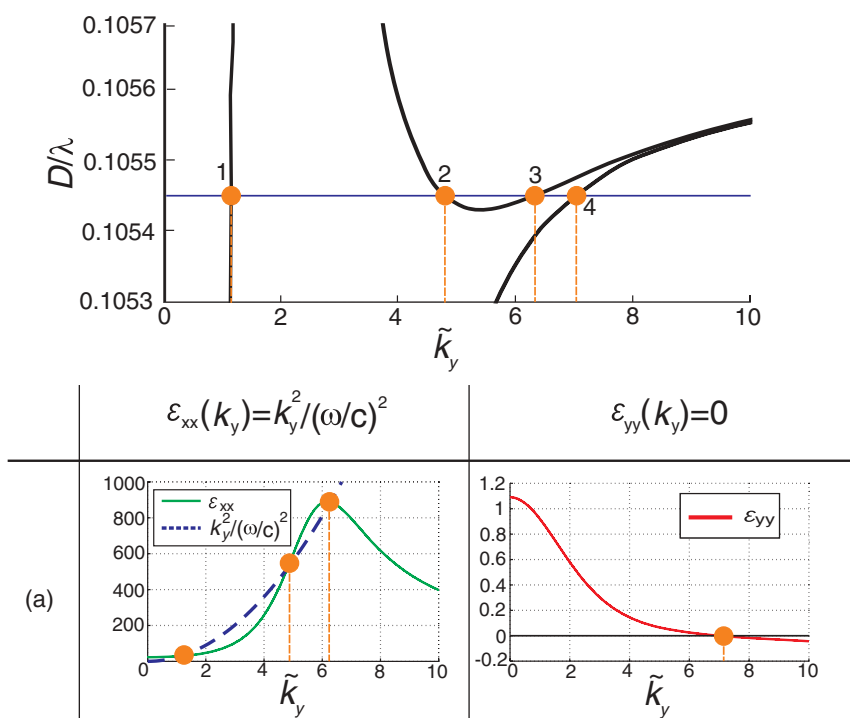

FIG. 4. (Color online) Fine structure of the dispersion curves of MLMMs in the vicinity of the plasmon resonance frequency and graphical solutions of dispersion equations (10) for $d_{1}=1.5 d_{2}$ and $D / \lambda=0.10545$. (a)

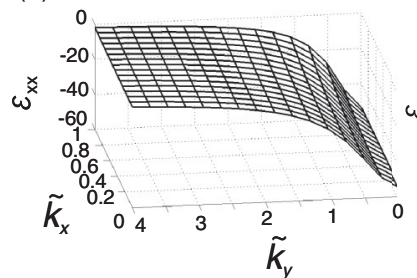

(b)

(c)

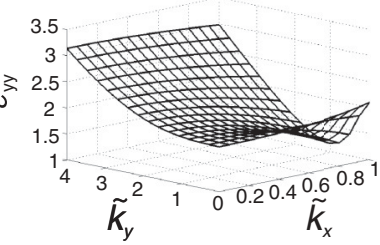

(d)

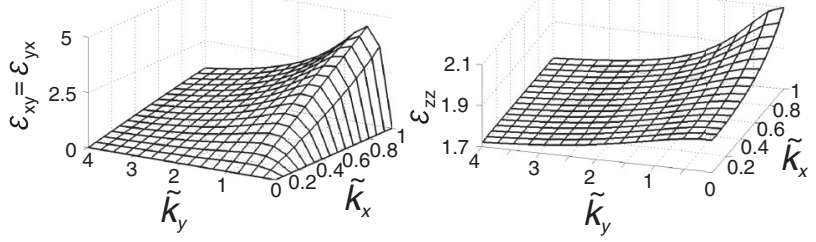

FIG. 5. Nonlocal permittivity tensor components: (a) $\varepsilon_{x x}$, (b) $\varepsilon_{y y}$, (c) $\varepsilon_{x y}, \varepsilon_{y x}$, (d) $\varepsilon_{z z}$ vs the normalized wave vector components $\tilde{k}_{x}=k_{x} D / \pi$ and $\tilde{k}_{y}=k_{y} D / \pi$ for metal-dielectric MLMMs at the wavelength $480 \mathrm{~nm}(D / \lambda=0.1302)$.

the presence or absence of the spatial dispersion features in the eigenwaves at a given frequency are also linked to the anisotropy of the corresponding permittivity tensor. If anisotropy of $\overline{\bar{\varepsilon}}(\omega, \mathbf{k})$ for a certain pair $(\omega, \mathbf{k})$ is sufficiently large (for the present structure, for $\left|\varepsilon_{x x} / \varepsilon_{y y}\right|>200$ ), one should expect a strong spatial dispersion at the frequency $\omega$.

\section{ANALYSIS OF SPATIALLY DISPERSIVE PERMITTIVITY}

Next, we analyze how the permittivity tensor of a metaldielectric MLMM depends on the wave vector at different frequencies. In Figs. 5-8 these dependencies are shown for MLMMs with the parameters $\varepsilon_{1}=4.6, \varepsilon_{2}=1-\lambda^{2} / \lambda_{p}^{2}, d_{1}=$ $37.5 \mathrm{~nm}$, and $d_{2}=25 \mathrm{~nm}, \lambda_{p}=250 \mathrm{~nm}$ at the wavelengths of $480 \mathrm{~nm}, 580 \mathrm{~nm}, 630 \mathrm{~nm}$, and $780 \mathrm{~nm}$, respectively.

As can be seen from Figs. 5(a) to 8(a), at the highest normalized frequency $D / \lambda=0.1302$ [see Fig. 5(a)] the component $\varepsilon_{x x}$ grows with $\tilde{k}_{y}$. At slightly lower frequency $D / \lambda=0.1078$, this component becomes a resonant function
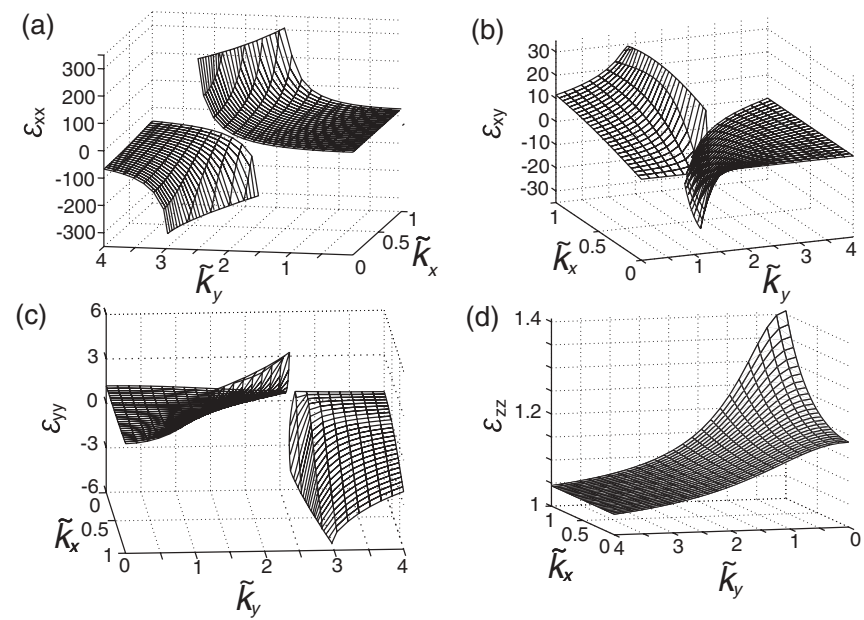

FIG. 6. The same as in Fig. 5 but at the wavelength $580 \mathrm{~nm}$ $(D / \lambda=0.1078)$. 


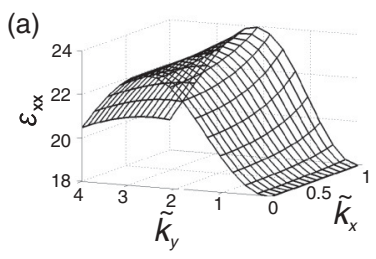

(c)

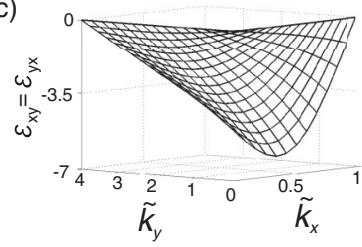

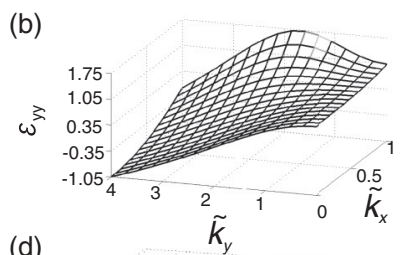

(d)

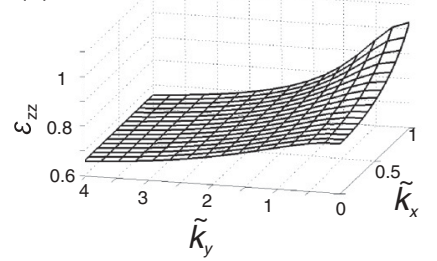

FIG. 7. The same as in Fig. 5 but at the wavelength $630 \mathrm{~nm}$ $(D / \lambda=0.0992)$.

of the wave vector [see Fig. 6(a)], and at $D / \lambda=0.0992$ it has a maximum [see Fig. 7(a)]. At the lowest frequency $D / \lambda=0.0801$ [see Fig. 8(a)], this component decreases with $\tilde{k}_{y}$. Resonant behavior of the tensor components can be described in terms of the permittivity pole,

$$
\left(\begin{array}{ll}
\varepsilon_{x x} & \varepsilon_{x y} \\
\varepsilon_{y x} & \varepsilon_{y y}
\end{array}\right)=\frac{1}{\left[\omega_{\text {res }}(\mathbf{k})-\omega\right]}\left(\begin{array}{ll}
A_{x x}(\omega, \mathbf{k}) & A_{x y}(\omega, \mathbf{k}) \\
A_{y x}(\omega, \mathbf{k}) & A_{y y}(\omega, \mathbf{k})
\end{array}\right),
$$

where $A_{\alpha \beta}$ are analytical functions. In the theory of continuous media this representation of the permittivity tensor (without its dependence on $\mathbf{k}$ ) has been obtained in the approximation of low optical losses and $\omega_{\text {res }}$ is the frequency of the resonant absorption. ${ }^{19}$ Our study also neglects losses, and we can interpret our $\tilde{\omega}_{\text {res }}=D / \lambda_{\text {res }}$ as the presumable frequency of resonant absorption if some small losses are introduced. This resonant absorption is of course related to the collective plasmon resonance of metal layers. However, due to the spatial dispersion this frequency depends on $\mathbf{k}$. We can see in Figs. 6(a)-6(c) that at the wavelength $580 \mathrm{~nm}$ three independent components of $\overline{\bar{\varepsilon}}$ are resonant $\left(\varepsilon_{x x}, \varepsilon_{y y}\right.$, and $\varepsilon_{x y}=$ $\left.\varepsilon_{y x}\right)$. Such behavior has been found in a rather wide range around $580 \mathrm{~nm}$ for all these components. In Fig. 9, we show the dispersion surface $\tilde{\omega}_{\text {res }}=D / \lambda_{\text {res }}$. A crossing of this surface with the dispersion diagram would deliver the frequencies
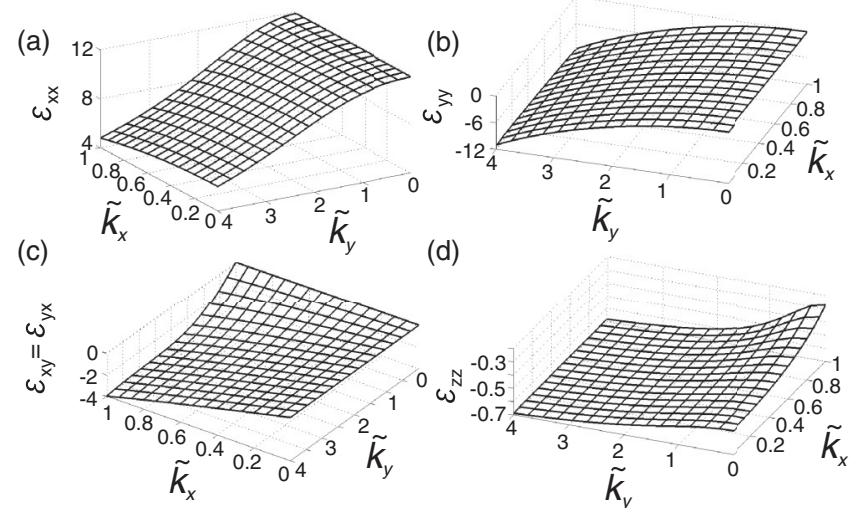

(d)

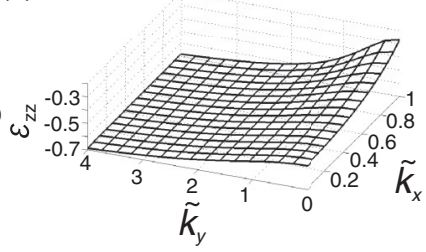

FIG. 8. The same as in Fig. 5 but at the wavelength $780 \mathrm{~nm}$ $(D / \lambda=0.0801)$.

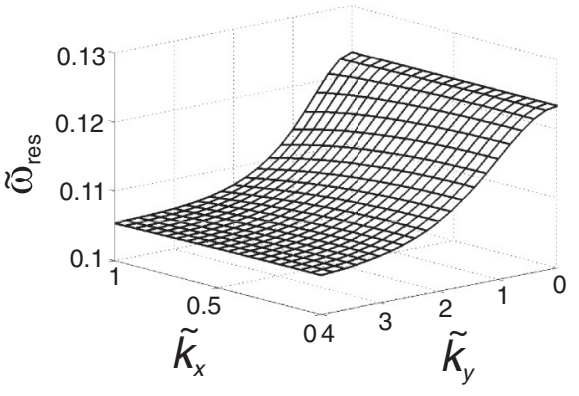

FIG. 9. Normalized resonance frequency $\tilde{\omega}_{\text {res }}=\omega_{\text {res }} D / 2 \pi c$ as a function of the components $\tilde{k}_{x}=k_{x} D / \pi$ and $\tilde{k}_{y}=k_{y} D / \pi$.

and propagation directions at which the resonant absorption holds. Briefly, we claim that the resonant frequency in metaldielectric MLMMs depends on the propagation direction.

The only nonresonant component of the permittivity tensor is $\varepsilon_{z z}$. As can be seen in Figs. $5(\mathrm{~d})$ to $8(\mathrm{~d})$, there are no qualitative changes in the behavior of this component in respect to the wave vector at different frequencies: The component $\varepsilon_{z z}$ decreases with an increase of $\tilde{k}_{y}$, but it grows with the increase of $\tilde{k}_{x}$.

Next, we calculate the variation of $\varepsilon_{x y}(\omega, \mathbf{k})$ when the coordinate system rotates around $z$. The purpose of this calculation is to eliminate the off-diagonal component of the permittivity tensor transforming the latter to the form of a tensor of a uniaxial medium. This is possible to do for any $k_{y}$ at a given frequency $\omega$ (whereas the wave vector component $k_{x}$ is related to $k_{y}$ via the dispersion equation). For the semi-infinite interval $k_{0}<k_{y}<\infty$ at every frequency, there exists a finite value $k_{y}$ at which the needed rotation angle $\theta$ is maximal. At the wavelength $480 \mathrm{~nm}$ the maximum of the function $\theta\left(k_{y}\right)$ for this semi-infinite interval of $k_{y}$ equals $8^{\circ} 56^{\prime}$. At $630 \mathrm{~nm}$ $\theta_{\max }=15^{\circ} 40^{\prime}$, and $\theta_{\max }=13^{\circ} 7^{\prime}$ at $780 \mathrm{~nm}$. For a half space of our MLMMs whose boundary is parallel to the layers, the eigenmodes with $k_{0}<k_{y}<\infty$ can be excited only by incident evanescent waves. Eigenmodes with $k_{y}<k_{0}$ can be excited by propagating plane waves, and for these eigenmodes the optical axis rotation turned out to be much smaller. For $k_{y}<k_{0}$, the values of $\theta_{\max }$ are equal to $46^{\prime}$ at the wavelength $480 \mathrm{~nm}$, to $30^{\prime}$ at $580 \mathrm{~nm}$, to $21^{\prime}$ at $630 \mathrm{~nm}$, and to $17^{\prime}$ at $780 \mathrm{~nm}$. We can conclude that for incident propagating waves this effect of spatial dispersion can be practically negligible. However, this refers only to one special geometry of the boundary. If the interface of the MLMM half space is orthogonal to the layers the eigenmodes $k_{0}<k_{y}<\infty$ can be excited by incident plane waves $k_{x}<k_{0}$, as well. In this case the component $k_{x}$ of the incident wave vector is preserved in the excited eigenmode and eigenmodes with $k_{x}<k_{0}$ and $k_{y}>k_{0}$ exist in the metal-dielectric MLMMs. For this geometry, the optical axis rotation is an important effect of spatial dispersion which holds for propagating incident waves as well.

\section{LAYERED DIELECTRIC STRUCTURES}

For comparison, we apply the model of the nonlocal effective medium to the case of all-dielectric MLMMs. As an example, we consider an all-dielectric structure created by the layers of two dielectric materials with $\varepsilon_{1}=1$ and $\varepsilon_{2}=4.6$ 

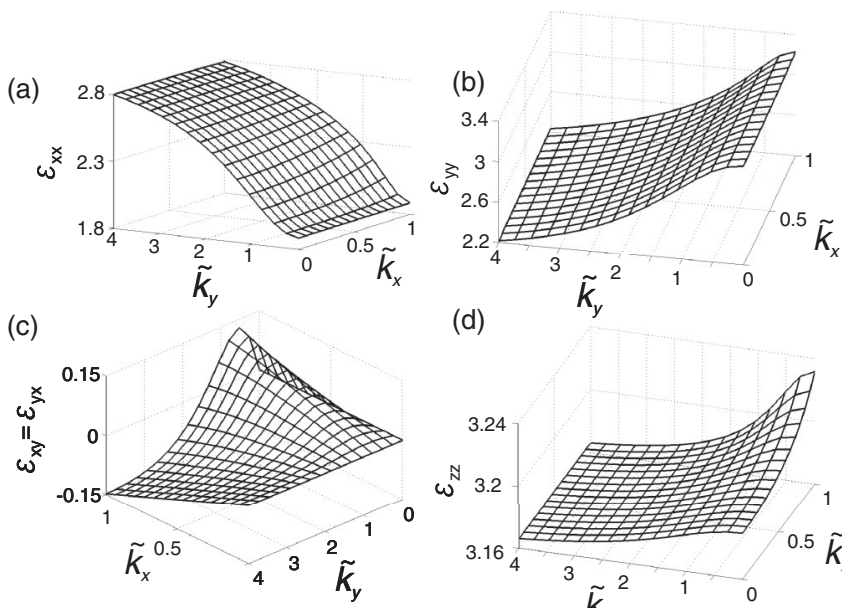

(d)

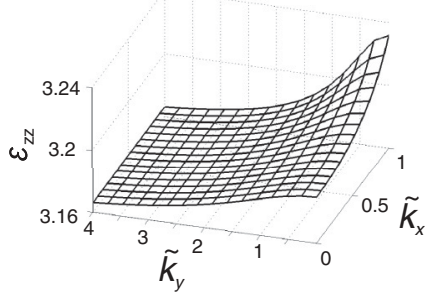

FIG. 10. Nonlocal permittivity tensor components: (a) $\varepsilon_{x x}$, (b) $\varepsilon_{y y}$, (c) $\varepsilon_{x y}, \varepsilon_{y x}$, (d) $\varepsilon_{z z}$ vs the normalized wave vector components $\tilde{k}_{x}=k_{x} D / \pi$ and $\tilde{k}_{y}=k_{y} D / \pi$ for all-dielectric layered structures with $\varepsilon_{1}=4.6$ and $\varepsilon_{2}=1$ at the wavelength of $580 \mathrm{~nm}\left(d_{1}=1.5 d_{2}\right)$.

at wavelength $580 \mathrm{~nm}$, also taking the layers' thicknesses as $d_{1}=37.5 \mathrm{~nm}$ and $d_{2}=25 \mathrm{~nm}$, respectively. The results are based on the analysis stated above, and they are presented in Fig. 10. From those results we observe that the components of the permittivity tensor vary noticeably along with variations of the wave vector even for this all-dielectric structure. Therefore, the spatial dispersion effects are still not negligible though they are not so strong as in the case of metal-dielectric MLMMs.

The component $\varepsilon_{x x}$ varies within $32 \%$ from its maximum value, and the component $\varepsilon_{y y}$ changes in the range of $31 \%$. In the case of all-dielectric multilayers, off-diagonal tensor components are also present, but their values range from -0.15 to 0.13 , depending on the wave vector. The smallest variation (no more than $2 \%$ ) is found for the component $\varepsilon_{z z}$.
Off-diagonal components $\varepsilon_{x y}$ and $\varepsilon_{y x}$ do not vanish, and they are quite noticeable. We calculate also the value of the angle $\theta_{\max }$ for the all-dielectric structure under consideration. At the wavelength $580 \mathrm{~nm}$ we obtain $\theta_{\max }=7^{\circ} 10^{\prime}$, for $k_{0}<$ $k_{y}<\infty$, and $\theta_{\max }=31^{\prime}$ for $k_{y}<k_{0}$.

\section{CONCLUSIONS}

We have studied the effects of spatial dispersion on the properties of nanostructured metal-dielectric metamaterials. We have verified the general concepts of the nonlocal homogenization theory earlier developed for such structures ${ }^{25,26}$ and also analyzed some novel effects associated with the manifestation of the nonlocal response. In particular, we have revealed that in nanostructured media the optical axis becomes tilted with respect to its position in the homogeneous dielectric medium, and this tilt depends on both frequency and propagation direction. We have shown numerically that this effect is significant not only for metal-dielectric metamaterials but also for all-dielectric nanostructured materials. We have also pointed out the correspondence between the extreme anisotropy in the nonlocal permittivity tensor and the spatial dispersion. Multilayered metamaterials provide a remarkable example of nanostructured materials for which the nonlocal homogenization theory can be developed and implemented analytically.

\section{ACKNOWLEDGMENTS}

This work has been supported by the Ministry of Education and Science of the Russian Federation, the Dynasty Foundation, EPSRC (UK), and the Australian Research Council.

\section{APPENDIX}

After some algebra, we rewrite the relations of the appendices of Ref. 25 in a more explicit form. The component orthogonal to the propagation plane can be written as follows:

$$
\begin{aligned}
\varepsilon_{z z}(\omega, \mathbf{k})= & {\left[\left(\varepsilon_{1} d_{1}\left(\varepsilon_{2} k_{0}^{2}-k^{2}\right)+\varepsilon_{2} d_{2}\left(\varepsilon_{1} k_{0}^{2}-k^{2}\right)\right)\left(\varepsilon_{1} k_{0}^{2}-k^{2}\right)\left(\varepsilon_{2} k_{0}^{2}-k^{2}\right) \Delta_{\mathrm{TE}}-2 \alpha\left(\varepsilon_{1}-\varepsilon_{2}\right)^{2} k_{0}^{2} k^{2}\right] } \\
& \times\left[\left(d_{1}\left(\varepsilon_{2} k_{0}^{2}-k^{2}\right)+d_{2}\left(\varepsilon_{1} k_{0}^{2}-k^{2}\right)\left(\varepsilon_{1} k_{0}^{2}-k^{2}\right)\right)\left(\varepsilon_{2} k_{0}^{2}-k^{2}\right) \Delta_{\mathrm{TE}}-2 \alpha\left(\varepsilon_{1}-\varepsilon_{2}\right)^{2} k_{0}^{4}\right]^{-1}, \\
\alpha= & 2 A+B\left(k_{x 1}^{2}+k_{x}^{2}\right)+C\left(k_{x 2}^{2}+k_{x}^{2}\right), \\
A= & k_{x 1} k_{x 2} k_{x}\left[\cos \left(k_{x 2} d_{2}\right) \sin \left(k_{x} d_{1}\right)+\cos \left(k_{x 1} d_{1}\right) \sin \left(k_{x} d_{2}\right)-\sin \left(k_{x} D\right)\right], \\
B= & k_{x 2} \sin \left(k_{x 1} d_{1}\right)\left[\cos \left(k_{x 2} d_{2}\right)-\cos \left(k_{x} d_{2}\right)\right], \\
C= & k_{x 1} \sin \left(k_{x 2} d_{2}\right)\left[\cos \left(k_{x 1} d_{1}\right)-\cos \left(k_{x} d_{1}\right)\right], \\
\Delta_{\mathrm{TE}}= & \sin \left(k_{x 1} d_{1}\right) \sin \left(k_{x 2} d_{2}\right)\left(k_{x 1}^{2}+k_{x 2}^{2}\right)+2 k_{x 1} k_{x 2}\left[\cos \left(k_{x} D\right)-\cos \left(k_{x 1} d_{1}\right) \cos \left(k_{x 2} d_{2}\right)\right],
\end{aligned}
$$

where $\mathbf{k}$ is the wave vector in the metamaterial, $k_{x 1}=\sqrt{\varepsilon_{1} k_{0}^{2}-k_{y}^{2}}, k_{x 2}=\sqrt{\varepsilon_{2} k_{0}^{2}-k_{y}^{2}}$.

The relations for other components look more compact in the matrix form:

$$
\left(\begin{array}{ll}
\varepsilon_{x x}(\omega, \mathbf{k}) & \varepsilon_{x y}(\omega, \mathbf{k}) \\
\varepsilon_{y x}(\omega, \mathbf{k}) & \varepsilon_{y y}(\omega, \mathbf{k})
\end{array}\right)=\langle\overline{\bar{E}}\rangle^{-1}\langle\overline{\bar{D}}\rangle .
$$


Components of the matrices $\langle\overline{\bar{E}}\rangle$ and $\langle\overline{\bar{D}}\rangle$ can be written in the following form:

$$
\begin{aligned}
& \langle\mathrm{E}\rangle_{x x}=\left[k_{x}^{2}\left(k_{0}^{2}\left(\varepsilon_{1}^{2} d_{2}+\varepsilon_{2}^{2} d_{1}\right)-k^{2}\left(\varepsilon_{1} d_{2}+\varepsilon_{2} d_{1}\right)\right)+\varepsilon_{1} \varepsilon_{2} k_{0}^{2}\left(k^{2} D-k_{0}^{2}\left(\varepsilon_{1} d_{2}+\varepsilon_{2} d_{1}\right)\right)\right]\left(\varepsilon_{1} \varepsilon_{2}\right)^{-1} \\
& +\frac{P k_{y}^{2}}{\varepsilon_{1} \varepsilon_{2}}\left\{2 A \varepsilon_{1} \varepsilon_{2} k_{0}^{2}\left[k_{0}^{2}\left(\varepsilon_{1}+\varepsilon_{2}\right)-k^{2}\right]-B \varepsilon_{1}\left[\varepsilon_{2}^{2} k_{0}^{4}\left(\varepsilon_{1} k_{0}^{2}-k_{y}^{2}\right)+k_{x}^{2}\left(k^{2}-k_{0}^{2}\left(\varepsilon_{1}+\varepsilon_{2}\right)\right)^{2}\right]\right. \\
& \left.-C \varepsilon_{2}\left[\varepsilon_{1}^{2} k_{0}^{4}\left(\varepsilon_{2} k_{0}^{2}-k_{y}^{2}\right)+k_{x}^{2}\left(k^{2}-k_{0}^{2}\left(\varepsilon_{1}+\varepsilon_{2}\right)\right)^{2}\right]\right\}, \\
& \langle\mathrm{E}\rangle_{x y}=\langle\mathrm{E}\rangle_{y x}=-k_{x} k_{y}\left[k^{2}\left(\varepsilon_{2} d_{1}+\varepsilon_{1} d_{2}\right)-k_{0}^{2}\left(\varepsilon_{2}^{2} d_{1}+\varepsilon_{1}^{2} d_{2}\right)\right]\left(\varepsilon_{1} \varepsilon_{2}\right)^{-1} \\
& -\frac{P k_{y}}{\varepsilon_{1} \varepsilon_{2}}\left\{A \frac{\varepsilon_{1} \varepsilon_{2} k_{0}^{2}}{k_{x}}\left[\varepsilon_{1} \varepsilon_{2} k_{0}^{4}+\left(k_{y}^{2}-k_{x}^{2}\right)\left(k^{2}-\left(\varepsilon_{1}+\varepsilon_{2}\right) k_{0}^{2}\right)\right]-B \varepsilon_{1} k_{x}\left[\varepsilon_{2}^{2} k_{0}^{4}\left(\varepsilon_{1} k_{0}^{2}-k_{y}^{2}\right)\right.\right. \\
& \left.\left.+\left[\varepsilon_{1} \varepsilon_{2} k_{0}^{4}+k_{y}^{2}\left(k^{2}-\left(\varepsilon_{1}+\varepsilon_{2}\right) k_{0}^{2}\right)\right]\left[\left(\varepsilon_{1}+\varepsilon_{2}\right) k_{0}^{2}-k^{2}\right)\right]\right]-C \varepsilon_{2} k_{x}\left[\varepsilon_{1}^{2} k_{0}^{4}\left(\varepsilon_{2} k_{0}^{2}-k_{y}^{2}\right)+\left(\left(\varepsilon_{1}+\varepsilon_{2}\right) k_{0}^{2}-k^{2}\right)\right. \\
& \left.\left.\times\left(k_{0}^{4} \varepsilon_{1} \varepsilon_{2}+k_{y}^{2}\left(k^{2}-\left(\varepsilon_{1}+\varepsilon_{2}\right) k_{0}^{2}\right)\right)\right]\right\}, \\
& \langle\mathrm{E}\rangle_{y y}=\left[k_{y}^{2}\left(k_{0}^{2}\left(\varepsilon_{1}^{2} d_{2}+\varepsilon_{2}^{2} d_{1}\right)-k^{2}\left(\varepsilon_{1} d_{2}+\varepsilon_{2} d_{1}\right)\right)+\varepsilon_{1} \varepsilon_{2} k_{0}^{2}\left(k^{2} D-k_{0}^{2}\left(\varepsilon_{1} d_{2}+\varepsilon_{2} d_{1}\right)\right)\right]\left(\varepsilon_{1} \varepsilon_{2}\right)^{-1} \\
& -\frac{P}{\varepsilon_{1} \varepsilon_{2}}\left\{2 A \varepsilon_{1} \varepsilon_{2} k_{0}^{2}\left[k_{y}^{2}\left(\left(\varepsilon_{1}+\varepsilon_{2}\right) k_{0}^{2}-k^{2}\right)-k_{0}^{4} \varepsilon_{1} \varepsilon_{2}\right]+B \varepsilon_{1}\left[\left(\varepsilon_{1} \varepsilon_{2} k_{0}^{4}+k_{y}^{2}\left(k^{2}-\left(\varepsilon_{1}+\varepsilon_{2}\right) k_{0}^{2}\right)\right)^{2}\right.\right. \\
& \left.\left.+\varepsilon_{2}^{2} k_{0}^{4} k_{x}^{2}\left(\varepsilon_{1} k_{0}^{2}-k_{y}^{2}\right)\right]+C \varepsilon_{2}\left[\varepsilon_{1}^{2} k_{0}^{4} k_{x}^{2}\left(\varepsilon_{2} k_{0}^{2}-k_{y}^{2}\right)+\left(\varepsilon_{1} \varepsilon_{2} k_{0}^{4}+k_{y}^{2}\left(k^{2}-\left(\varepsilon_{1}+\varepsilon_{2}\right) k_{0}^{2}\right)\right)^{2}\right]\right\}, \\
& \langle\mathrm{D}\rangle_{x x}=k_{0}^{2}\left[k_{x}^{2}\left(\varepsilon_{2} d_{1}+\varepsilon_{1} d_{2}\right)+k^{2}\left(\varepsilon_{1} d_{1}+\varepsilon_{2} d_{2}\right)\right]-D\left(k_{0}^{4} \varepsilon_{1} \varepsilon_{2}+k_{x}^{2} k^{2}\right)-P k_{y}^{2}\left\{A\left[-\varepsilon_{1} \varepsilon_{2} k_{0}^{4}+\left(\varepsilon_{1}+\varepsilon_{2}\right) k^{4}-k^{2} k_{0}^{2}\right]\right. \\
& \left.+B k_{0}^{2}\left[\varepsilon_{1} k_{x}^{2}\left(\varepsilon_{1} k_{0}^{2}-k^{2}\right)+\varepsilon_{2}\left(\varepsilon_{1} k_{0}^{2}\left(2 k_{x}^{2}+k_{y}^{2}\right)-k_{y}^{2} k^{2}\right)\right]+C k_{0}^{2}\left[\varepsilon_{2} k_{x}^{2}\left(\varepsilon_{2} k_{0}^{2}-k^{2}\right)+\varepsilon_{1}\left(\varepsilon_{2} k_{0}^{2}\left(2 k_{x}^{2}+k_{y}^{2}\right)-k_{y}^{2} k^{2}\right)\right]\right\}, \\
& \langle\mathrm{D}\rangle_{x y}=k_{x} k_{y}\left(k_{0}^{2}\left(\varepsilon_{2} d_{1}+\varepsilon_{1} d_{2}\right)-k^{2} D\right)-P k_{y}\left\{\frac{A}{k_{x}}\left[k_{y}^{2} k^{2}\left(k^{2}-\varepsilon_{2} k_{0}^{2}\right)+\varepsilon_{1}\left(\varepsilon_{2} k_{0}^{4}\left(k^{2}+k_{x}^{2}\right)-k^{2} k_{0}^{2} k_{y}^{2}\right)\right]\right. \\
& -B k_{0}^{2} k_{x}\left[\varepsilon_{1} k_{y}^{2}\left(k^{2}-\varepsilon_{1} k_{0}^{2}\right)+\varepsilon_{2}\left(\varepsilon_{1} k_{0}^{2}\left(k_{x}^{2}+\varepsilon_{1} k_{0}^{2}\right)-k_{y}^{2} k^{2}\right)\right]-C k_{0}^{2} k_{x}\left[\varepsilon_{2} k_{y}^{2}\left(k^{2}-\varepsilon_{2} k_{0}^{2}\right)\right. \\
& \left.\left.+\varepsilon_{1}\left(\varepsilon_{2} k_{0}^{2}\left(k_{x}^{2}+\varepsilon_{2} k_{0}^{2}\right)-k_{y}^{2} k^{2}\right)\right]\right\}, \\
& \langle\mathrm{D}\rangle_{y x}=k_{x} k_{y}\left(k_{0}^{2}\left(\varepsilon_{2} d_{1}+\varepsilon_{1} d_{2}\right)-k^{2} D\right)+P k_{x} k_{y}\left\{A\left[-\varepsilon_{1} \varepsilon_{2} k_{0}^{4}+\left(\varepsilon_{1}+\varepsilon_{2}\right) k^{4}-k^{2} k_{0}^{2}\right]\right. \\
& +B k_{0}^{2}\left[\varepsilon_{1} k_{x}^{2}\left(\varepsilon_{1} k_{0}^{2}-k^{2}\right)+\varepsilon_{2}\left(\varepsilon_{1} k_{0}^{2}\left(2 k_{x}^{2}+k_{y}^{2}\right)-k_{y}^{2} k^{2}\right)\right]+C k_{0}^{2}\left[\varepsilon_{2} k_{x}^{2}\left(\varepsilon_{2} k_{0}^{2}-k^{2}\right)\right. \\
& \left.\left.+\varepsilon_{1}\left(\varepsilon_{2} k_{0}^{2}\left(2 k_{x}^{2}+k_{y}^{2}\right)-k_{y}^{2} k^{2}\right)\right]\right\}, \\
& \langle\mathrm{D}\rangle_{y y}=k_{0}^{2}\left[k_{y}^{2}\left(\varepsilon_{2} d_{1}+\varepsilon_{1} d_{2}\right)+k^{2}\left(\varepsilon_{1} d_{1}+\varepsilon_{2} d_{2}\right)\right]-D\left(\varepsilon_{1} \varepsilon_{2} k_{0}^{4}+k_{y}^{2} k^{2}\right) \\
& +P k_{x}\left\{\frac{A}{k_{x}}\left[k_{y}^{2} k^{2}\left(k^{2}-\varepsilon_{2} k_{0}^{2}\right)+\varepsilon_{1}\left(\varepsilon_{2} k_{0}^{4}\left(k^{2}+k_{x}^{2}\right)-k^{2} k_{0}^{2} k_{y}^{2}\right)\right]-B k_{0}^{2} k_{x}\left[\varepsilon_{1} k_{y}^{2}\left(k^{2}-\varepsilon_{1} k_{0}^{2}\right)\right.\right. \\
& \left.\left.+\varepsilon_{2}\left(\varepsilon_{1} k_{0}^{2}\left(k_{x}^{2}+\varepsilon_{1} k_{0}^{2}\right)-k_{y}^{2} k^{2}\right)\right]-C k_{0}^{2} k_{x}\left[\varepsilon_{2} k_{y}^{2}\left(k^{2}-\varepsilon_{2} k_{0}^{2}\right)+\varepsilon_{1}\left(\varepsilon_{2} k_{0}^{2}\left(k_{x}^{2}+\varepsilon_{2} k_{0}^{2}\right)-k_{y}^{2} k^{2}\right)\right]\right\},
\end{aligned}
$$

where

$$
P=\frac{2\left(\varepsilon_{1}-\varepsilon_{2}\right)^{2} \Delta_{\mathrm{TM}}^{-1}}{\left(k^{2}-\varepsilon_{1} k_{0}^{2}\right)\left(k^{2}-\varepsilon_{2} k_{0}^{2}\right)} .
$$

The physcial meaning of $\langle\overline{\bar{E}}\rangle$ and $\langle\overline{\bar{D}}\rangle$ is the following: These two matrices describe, respectively, the components of the electric field and electric displacement field, normalized by the factor $i\left[\omega \varepsilon_{0}\left(k^{2}-\varepsilon_{1} k_{0}^{2}\right)\left(k^{2}-\varepsilon_{2} k_{0}^{2}\right)\right]^{-1}$ and averaged over the period of the structure, $D=d_{1}+d_{2}$.

${ }^{1}$ J. B. Pendry and S. A. Ramakrishna, Physica B 338, 329 (2003).

${ }^{2}$ P. A. Belov and Y. Hao, Phys. Rev. B 73, 113110 (2006).

${ }^{3}$ A. Salandrino and N. Engheta, Phys. Rev. B 74, 075103 (2006).

${ }^{4}$ J. Zubin, L. Alekseyev, and E. Narimanov, Opt. Express 14, 8247 (2006).
${ }^{5}$ Y. Xiong, Z. Liu, and X. Zhang, Appl. Phys. Lett. 93, 111116 (2008).

${ }^{6}$ J. Pendry, D. Schurig, and D. Smith, Science 3, 1 (2006).

${ }^{7}$ W. Cai, U. Chettiar, A. Kildishev, and V. Shalaev, Nature (London) 10, 1038 (2007).

${ }^{8}$ D. R. Smith and D. Schurig, Phys. Rev. Lett. 90, 077405 (2003). 
${ }^{9}$ E. Purcell, Phys. Rev. 69, 681 (1946).

${ }^{10}$ P. Yao, C. Van Vlack, A. Reza, M. Patterson, M. M. Dignam, and S. Hughes, Phys. Rev. B 80, 195106 (2009).

${ }^{11}$ H. Xie, P. Leung, and D. Tsai, Solid State Commun. 149, 625 (2009).

${ }^{12}$ O. Kidwai, S. Zhukovsky, and J. Sipe, Opt. Lett. 36, 2530 (2011).

${ }^{13}$ A. N. Poddubny, P. A. Belov, and Yu. S. Kivshar, Phys. Rev. A 84, 023807 (2011).

${ }^{14}$ I. Iorsh, A. Poddubny, A. Orlov, P. Belov, and Y. Kivshar, Phys. Lett. A 376, 185 (2012).

${ }^{15}$ S. Rytov, J. Exp. Theor. Phys. 2, 466 (1956).

${ }^{16}$ A. Vinogradov and A. Merzlikin, J. Exp. Theor. Phys. 94, 482 (2002).

${ }^{17}$ J. Elser, A. A. Govyadinov, I. Avrutsky, I. Salakhutdinov, and V. Podolskiy, J. Nanomater. 2007, 79469 (2007).

${ }^{18}$ J. Elser, V. Podolskiy, I. Salakhutdinov, and I. Avrutsky, Appl. Phys. Lett. 90, 191109 (2007).
${ }^{19}$ L. Landau, L. Pitaevskii, and E. Lifshitz, Electrodynamics of Continuous Media (Butterworth-Heinemann, Oxford, 1984).

${ }^{20}$ V. Agranovich and V. Ginzburg, Crystal Optics with Spatial Dispersion and Excitons (Springer-Verlag, Berlin, 1984).

${ }^{21}$ P. A. Belov and C. R. Simovski, Phys. Rev. E 72, 026615 (2005).

${ }^{22}$ P. A. Belov, R. Marques, S. I. Maslovski, I. S. Nefedov, M. Silveirinha, C. R. Simovski, and S. A. Tretyakov, Phys. Rev. B 67, 113103 (2003).

${ }^{23}$ G. Castaldi, V. Galdi, A. Alu, and N. Engheta, Phys. Rev. Lett. 108, 063902 (2012).

${ }^{24}$ M. G. Silveirinha, Phys. Rev. B 75, 115104 (2007).

${ }^{25}$ A. V. Chebykin, A. A. Orlov, A. V. Vozianova, S. I. Maslovski, Yu. S. Kivshar, and P. A. Belov, Phys. Rev. B 84, 115438 (2011).

${ }^{26}$ A. A. Orlov, P. M. Voroshilov, P. A. Belov, and Yu. S. Kivshar, Phys. Rev. B 84, 045424 (2011).

${ }^{27}$ A. P. Vinogradov, A. V. Dorofeenko, and I. A. Nechepurenko, Metamaterials 4, 181 (2010). 